\title{
Testing the Therapeutic Equivalence of Alogliptin, Linagliptin, Saxagliptin, Sitagliptin or Vildagliptin as Monotherapy or in Combination with Metformin in Patients with Type 2 Diabetes
}

\author{
Andrea Messori • Valeria Fadda $\cdot$ Dario Maratea \\ Sabrina Trippoli $\cdot$ Claudio Marinai
}

To view enhanced content go to www.diabetestherapy-open.com

Received: March 29, 2014 / Published online: May 3, 2014

(c) The Author(s) 2014. This article is published with open access at Springerlink.com

\begin{abstract}
Background: In studying the therapeutic evidence of innovative drug treatments, increasing attention is being devoted to differentiating between results that indicate no significant differences among the treatments under examination ("no proof of difference") and results that demonstrate the therapeutic equivalence among the treatments ("proof of no difference").
\end{abstract}

Aim: Our analysis was aimed at evaluating the degree of therapeutic equivalence for dipeptidylpeptidase-4 (DPP-4) inhibitors given in type 2 diabetes as monotherapy or in combination with metformin.

Methods: Equivalence was determined by developing a standard Forest plot that incorporated the information on margins

Electronic supplementary material The online version of this article (doi:10.1007/s13300-014-0066-y) contains supplementary material, which is available to authorized users.

A. Messori $(\bowtie) \cdot$ V. Fadda $\cdot$ D. Maratea .

S. Trippoli · C. Marinai

HTA Unit, ESTAV Toscana Centro, Regional Health

Service, 50100 Florence, Italy

e-mail: andrea.messori.it@gmail.com;

andrea.messori@estav-centro.toscana.it previously reported in randomized trials on these agents. The end point was $\mathrm{HbA}_{1 \mathrm{c}}$ change from baseline; the equivalence margin was set at $\pm 0.25 \%$ change in $\mathrm{HbA}_{1 \mathrm{c}}$. The clinical material was obtained from a systematic review on this topic.

Results: Given as monotherapy, linagliptin, sitagliptin, and vildagliptin (but not saxagliptin) met the equivalence criterion when compared with one another. Given in combination with metformin, linagliptin, saxagliptin, sitagliptin, and vildagliptin showed an equivalent effect whereas alogliptin did not satisfy the equivalence criterion.

Conclusions: Considering the most recent therapeutic guidelines, our results are of interest particularly as regards the information on DPP-4 inhibitors in combination with metformin. Four of the five DPP-4 inhibitors under examination clearly showed to have the same effectiveness; the fifth agent-alogliptinfailed to meet the equivalence criterion, but only because its superiority could not be excluded.

Keywords: Dipeptidylpeptidase-4 inhibitors; DPP-4; Equivalence; Meta-analysis; Type 2 diabetes 
In studying the therapeutic evidence of innovative drug treatments, increasing attention is being devoted to differentiating between results that indicate no significant differences among the treatments under examination ("no proof of difference") and results that demonstrate the therapeutic equivalence among the treatments ("proof of no difference"). It is well known that this latter conclusion is more informative than the former because "proof of no difference" is a conclusive result whereas "no proof of difference" is an inconclusive one [1-3].

No such analysis has thus far been conducted concerning dipeptidylpeptidase-4 (DPP-4) inhibitors that represent a pharmacological class increasingly employed in patients with type 2 diabetes.

In this study, we re-analyzed the clinical material presented in the most recent and most exhaustive systematic review in this area [4] to quantify the degree of therapeutic equivalence for DPP-4 inhibitors given in this disease condition as monotherapy or in combination with metformin.

Our clinical material included the results of all direct and indirect meta-analytical comparisons that Craddy and co-workers [4] have published in their recent systematic review on this topic. Testing equivalence is particularly straightforward when the information on margins is combined with standard Forest plots [5, 6]. Margins represent a threshold between clinically relevant incremental benefits and irrelevant ones, and can be retrieved from statistical power information of original trials.

We have applied this approach to evaluate the equivalence of monotherapy with alogliptin, linagliptin, saxagliptin, sitagliptin or vildagliptin versus placebo (Fig. 1a) and the equivalence of the same agents in combination with metformin versus metformin alone (Fig. 1b). The end point was $\mathrm{HbA}_{1 \mathrm{c}}$ change from baseline. The equivalence margins were set at $\pm 0.25 \%$ change in $\mathrm{HbA}_{1 \mathrm{c}}$ according to Buse et al. [7]. The clinical material that was reported by Craddy et al. [4] is presented in their Table 5.

In our first analysis (Fig. 1a), our equivalence testings found an equivalent treatment effect for linagliptin, sitagliptin, and vildagliptin given as monotherapy when compared with one another; the effect of saxagliptin did not satisfy the equivalence criterion, while the result for alogliptin was borderline. In our second analysis (Fig. 1b), the combination of the same five agents in comparison with metformin alone showed an equivalent effect for linagliptin, saxagliptin sitagliptin, and vildagliptin whereas the combination including alogliptin did not satisfy the equivalence criterion.

It should be kept in mind that, in patients with type 2 diabetes, all international guidelines advocate metformin first. Hence, our analysis on monotherapy with DPP-4 inhibitors had more speculative than a practical interest. In contrast, the analysis evaluating the combination of DPP-4 inhibitors with metformin had some practical interest. In fact, four of the five DPP-4 inhibitors clearly showed to have the same effectiveness; the fifth agent-alogliptin-failed to meet the less important criterion of the "left" margin (so that its superiority cannot be excluded), but, however, fully satisfied the criterion of being non-inferior in comparison with the "right" margin.

While the safety of these drugs is another important factor for defining their respective role in comparative terms, the evidence on this point seems to be more difficult to interpret because of the diversity of the safety end points and their relatively low frequency of occurrence [4]. 

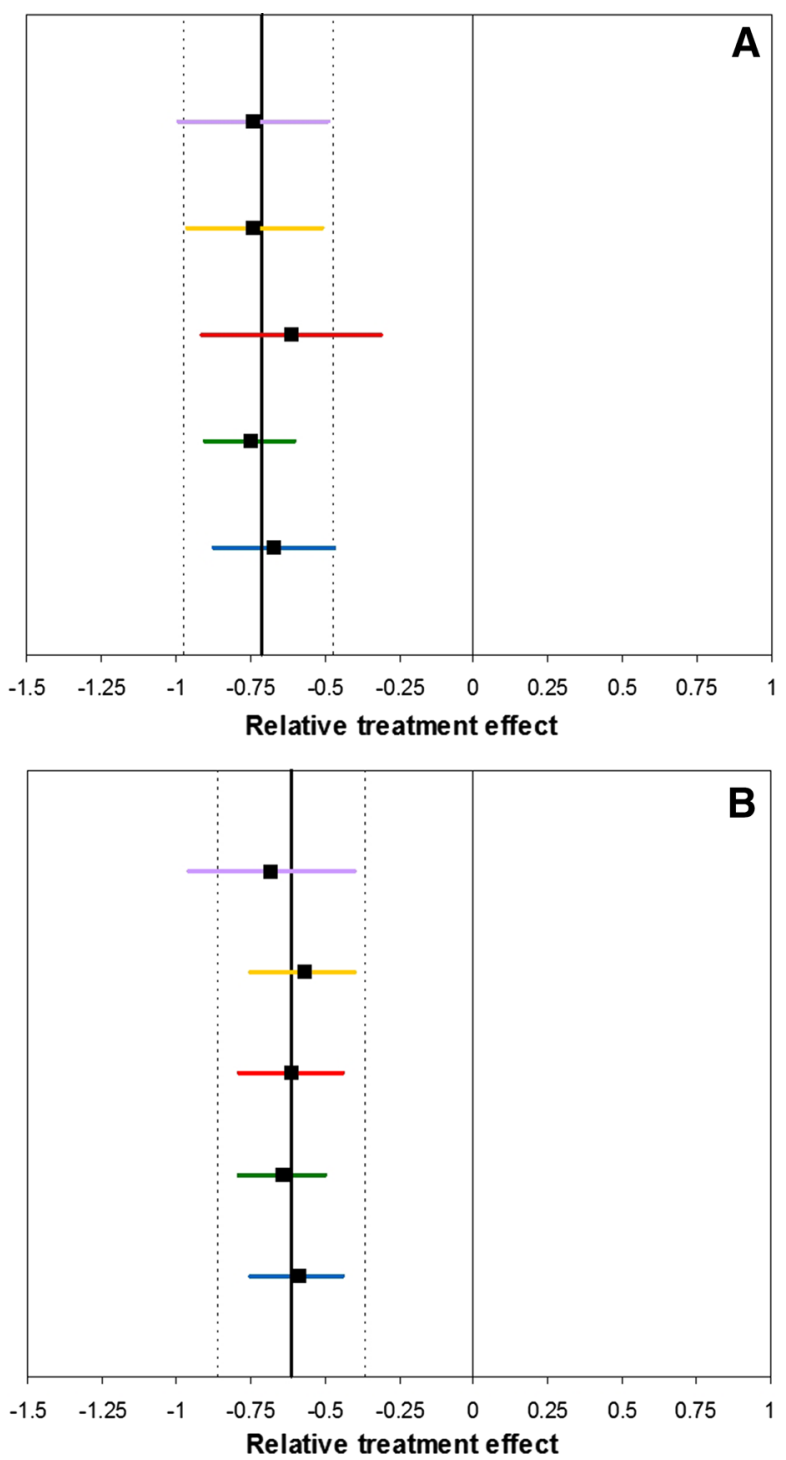

Fig. 1 Forest plot with equivalence margins: relative treatment effect of five DDP4 inhibitors evaluated as monotherapy versus placebo (a) or as combination with metformin versus metformin alone (b). Incremental effectiveness is expressed according to the end point of $\mathrm{HbA}_{1 \mathrm{c}}$ change from baseline. In both panels, the horizontal bars (alogliptin in violet, linagliptin in yellow, saxagliptin in red, sitagliptin in green, vildagliptin in blue) indicate the two-sided $95 \%$ confidence interval for the meta-analytic estimate (solid square) of relative treatment effect. The equivalence interval is comprised between the two vertical

In conclusion, although our analyses have entirely been based on the same clinical material already published by Craddy et al. [4], dotted lines and is centered around the value of weighted incremental effectiveness (vertical solid line) across the five agents $(-0.71$ in $\mathbf{a},-0.61$ in $\mathbf{b})$. The equivalence test is applied based on the equivalence intervals that reflect the margins reported by Buse et al. [7]. The criterion for demonstrating equivalence (at alpha level $=2.5 \%$ ) is when the entire $95 \%$ confidence interval remains within the equivalence interval. $P$ values for equivalence were: a $(t o p$ to bottom) $0.038,0.024,0.177,0.002,0.023 ; \mathbf{b}$ (top to bottom) 0.103, 0.01, 0.003, 0.001, 0.002

our results convey original information to better interpret the effectiveness of these agents in terms of equivalence. When 
treatment with a DPP-4 inhibitor in combination with metformin is started in a given patient, our findings indicate that the magnitude of the reduction in $\mathrm{HbA}_{1 \mathrm{c}}$ cannot represent the main criterion for selecting a specific agent in a given patient, since the expected improvement is essentially the same across these agents. Other criteria should, therefore, prevail, including the dosing schedule, the profile of adverse effects, and, last but not least, the cost.

\section{ACKNOWLEDGMENTS}

No funding or sponsorship was received for this report or publication of this article. All named authors meet the ICMJE criteria for authorship for this manuscript, take responsibility for the integrity of the work as a whole, and have given final approval for the version to be published.

Conflict of interest. Andrea Messori, Valeria Fadda, Dario Maratea, Sabrina Trippoli and Claudio Marinai declare no conflict of interest.

Compliance with ethics. The analysis in this article is based on previously conducted studies, and does not involve any new studies of human or animal subjects performed by any of the authors.

Open Access. This article is distributed under the terms of the Creative Commons Attribution Noncommercial License which permits any noncommercial use, distribution, and reproduction in any medium, provided the original author(s) and the source are credited.

\section{REFERENCES}

1. Messori A, Fadda V, Gatto R, Maratea D, Trippoli S. Differentiating between "no proof of difference" and "proof of no difference" for new oral anticoagulants. BMJ. 2014;6(348):g1955. doi:10.1136/bmj.g1955.

2. Messori A, Fadda V, Maratea D, Trippoli S. Rates of inhibitor development in previously untreated patients with severe hemophilia a treated with plasma-derived or recombinant Factor VIII: no proof of difference or proof of no difference? Semin Thromb Hemost. 2014;40(2):269-70.

3. Messori A, Fadda V, Maratea D, Trippoli S. Erythropoietin in patients with acute myocardial infarction: no proof of effectiveness or proof of no effectiveness? Clin Cardiol. 2013;36(10):E39-40.

4. Craddy P, Palin HJ, Johnson KI. Comparative effectiveness of dipeptidylpeptidase- 4 inhibitors in type 2 diabetes: a systematic review and mixed treatment comparison. Diabetes Ther. 2014 (Epub ahead of print).

5. Ahn S, Park SH, Lee KH. How to demonstrate similarity by using noninferiority and equivalence statistical testing in radiology research. Radiology. 2013;267(2):328-38.

6. Christensen E. Methodology of superiority vs. equivalence trials and non-inferiority trials. J Hepatol. 2007;46(5):947-54.

7. Buse JB, Nauck M, Forst T, Sheu WH, Shenouda SK, Heilmann CR, Hoogwerf BJ, Gao A, Boardman MK, Fineman M, Porter L, Schernthaner G. Exenatide once weekly versus liraglutide once daily in patients with type 2 diabetes (DURATION-6): a randomised, openlabel study. Lancet. 2013;381(9861):117-24. 